\title{
Perceived Organizational Support: A Literature Review
}

\author{
Li Sun (corresponding author) \\ Lecturer, School of Foreign Languages, Nanyang Institute of Technology \\ No. 80 Changjiang Road, Nanyang 473004, China \\ E-mail: sunli_lizhi@163.com
}

Received: June 12, 2019 Accepted: July 10, 2019 Online published: July 15, 2019

doi:10.5296/ijhrs.v9i3.15102 URL: https://doi.org/10.5296/ijhrs.v9i3.15102

\begin{abstract}
Perceived organizational support (POS), which reflects the organization's value on employees' contributions and concern for employee benefits, is an important tool to help organizations establish favourable relationship with employees and motivate employees work hard. This paper aims to make a review of POS concept and the variables related to POS. Research results showed that perceived organizational support emphasizes organizational commitment to employees and is a unilateral relationship. Perceived organizational support is related to employees' reduced absenteeism, increased job satisfaction, happiness, organizational citizenship behaviour and organizational performance, and also influenced by organizational fairness, working conditions, HR practices, employee characteristics, leadership, management communication. Besides, this paper summarized the measures to improve perceived organizational support, such as supportive or high commitment HR practices, fairness, superior and co-worker supports, employee empowerment. This study helps researchers understand the latest research progress in perceived organizational support.
\end{abstract}

Keywords: perceived organizational support, literature review, recommendations

\section{Introduction}

According to human capital theory (Becker, 2002), human capital (the knowledge, information, ideas, skills, and health of individuals) was by far the most important form of capital in modern economies. The main source in any company was the people, and the most successful companies would be those that manage human capital more effectively, by investing in their employees, encouraging workers to invest in themselves, providing learning environments. However, due to economic globalization, the cases of company failures, restructurings, mergers and layoffs around the world have had a huge impact on the work enthusiasm of employees in the organization and caused employee mistrust of their organization. Under such circumstances, how to maintain favorable relationship with 
employees is very critical for the organization to motivate employees to be dedicated to the organization and then increase organizational performance.

Aselage and Eisenberger (2003) stated that when an individual accepted the "positive treatment" of others, it naturally generated the psychological pressure and sense of obligation to pay off, thereby generating an attitude or behavior of retribution to the one given. When applied to the organizational environment, employees often give more positive feedback when they perceive the organization's concern and appreciation and affirmation. Therefore, this paper aims to make a review of the concept "perceived organizational support" which reflects employee-organizational relationship from the perspective of employee perception and which have been proved to have important benefits for employees and employers. For example, studies have found that employees with a strong exchange ideology reciprocated perceived organizational support by altering their efforts to meet organizational goals, such as reduce absenteeism, increase affective attachment to the organization and work effort (Eisenberger et al., 1986). In addition, perceived organizational support positively relates to employees' happiness (Kurtessi et al., 2015), job satisfaction (McCarthy et al., 2013), entrepreneurial passion (Gao \& Yan), employee performance (Shoss et al., 2013), organizational performance (Eisenberger \& Stinglhamber, 2011).

\section{Perceived Organizational Support and Psychological Contracts}

Perceived organizational support is an employee perception that the organization values their contributions and cares about their well-being (Eisenberger et al., 1986). After Eisenberger et al. proposed the concept of perceived organizational support, subsequent scholars mainly focused on the development of measurement scale, the factors affecting perceived organizational support, and the positive impact of perceived organizational support on employees and organizations through empirical studies.

A psychological contract is considered as the set of individual expectations about the employment relationship (Schermerhorn, 2010). Perceived organizational support is easily confused with psychological contracts. Studies have shown that perceived organizational support is an independent concept and has some similarities with and differences from psychological contracts. In terms of similarities between the two concepts. First, perceived organizational support satisfies the emotional needs of employees and enables employees to have a sense of responsibility and sentiment towards the organization; Likewise, the satisfaction of psychological contract also makes employees produce the sense of responsibility for the organization. Second, perceived organizational support and psychological contract mean that if employees think the goodness they get is from the voluntary behavior of the organization, not the outside pressures, such as trade union negotiations and government regulations, employees tend to generate higher evaluation to the organization (Eisenberger et al., 1997). Third, both organizational support theory and psychological contract theory apply social exchange theory to the organizational context and discuss the social exchange between employees and employers and use the principle of reciprocity as the explanation for the outcome variables (attitudinal and behavioral variables).

However, the two concepts also have some distinctions. First, psychological contract relies on 
mutual commitment between employees and organizations and represents the perception of employees and employers on reciprocal obligations. On the contrary, perceived organizational support emphasizes the organization's commitment to employees and is therefore unilateral. Second, the sense of organizational support is generally generated after employees enter the organization through their own observations and the related behaviors of superiors, while psychological contracts may be generated before employees enter the organization (Xu \& Zhang, 2005).

\section{Measurement of Perceived Organizational Support}

\subsection{One-dimensional Scale}

The earliest researcher who studied perceived organizational support was Eisenberger et al. (1986) who stated that perceived organizational support was a one-dimensional construct and developed an organizational support measure scale with a total of 36 items. After factor analysis, Eisenberger et al. (1986) found that the scale supported one-dimensional conception and had good reliability. This scale was the most widely used measure of organizational support in subsequent studies. However, in the specific application, follow-up researchers often selected some of 36 items in order to constitute a separate scale to make their study more convenient. This also reflects that the scale of 36 items developed by Eisenberger et al. (1986) has a high internal validity and a good one-dimensional character.

\subsection{Two-dimensional Scale}

McMillin (1997) supplemented the concept proposed by Eisenberger, and stated that organizational support should include, in addition to the organization's concern and respect for employees, tool support such as equipment, information, personnel, etc., necessary for the job staff to work better. The author proposed that organizational support should be composed of social emotional support and tool support and developed a 15-item measurement scale.

\subsection{Multiple-dimensional Scale}

Kraimer and Wayne (2004) divided the sense of organizational support into three dimensions: adaptive support, career support and financial support in the process of studying the sense of organizational support of expatriates, developed a 12-item scale. Ling et al. (2006) divided perceived organizational support into three dimensions: value recognition, job support and care about interests, and developed a 24-item scale to measure the three dimensions by use of Likert-6 scale, obtained high reliability and validity. Chen (2006) four-dimension scale (16 items) and nine-dimension scale (56 items) of perceived organizational support. The four-dimension scale includes emotional support, instrumental support, superior support and colleague support. Nine-dimension scale includes work support, value, competence and ambition, interpersonal support, welfare and job security, tolerance and understanding, fair and reasonable support, concern for respect, career development. Yuan (2017) developed a five-dimension scale of perceived organizational support. The five dimensions are respectively job support, value recognition, care about interests, emotional support and developmental support with a total of 23 measurement items. 


\section{Antecedents and Outcomes of Perceived Organizational Support}

\subsection{Antecedent Variables of Perceived Organizational Support}

The antecedent variables that affect perceived organizational support can be summarized as three categories, including organizational factors, individual factors, relationship between organization or organizational agents and employees (see Table 1). Among them, the organizational factors include organizational fairness, working conditions, organizational political activities, organizational size, organizational cultures. Individual factors include values, positive or negative emotions, pre-hiring experience, work status and other variables. Relationship between organization or organizational agents and employees include psychological contract, employee-organization match, leader-member exchange, management communication, leadership style and other variables.

\subsubsection{Organizational Factors}

The results of Wayne et al. (2002) showed that organizational justice, managerial tolerance and recognition were related to perceived organizational support. Ling et al. (2006) stated that procedural fairness affected the formation of perceived organizational support. Ghani (2009) pointed out that trust, access to information, learning and development opportunities were positively related to perceived organizational support. Trust was the most effective variable predicting organizational support. DeConinck (2010) considered distributive fairness was an antecedent variable of perceived organizational support. Dawley et al. (2010) confirmed that perceived supervisory support was a variable predicting perceived organizational support. Meta-analysis of Ahmed and Nawaz (2015) revealed that perceived organizational support was largely influenced by fairness, growth opportunities, supervisory support, and colleague support. Chen et al. (2017) stated that supportive HR practices had a positive impact on perceived organizational support. Yu et al. (2017) stated visionary leadership had an impact on perceived organizational support. Xue (2017) stated that perceived organizational justice had a significant impact on perceived organizational support. Lin (2018) stated that "career development" and "performance evaluation" in developmental human resource practices had positive impact on perceived organizational support. Zhang (2018) verified that organizational socialization strategy had a significantly positive impact on perceived organizational support, and stated that the better the organization socialization strategy is implemented, the higher the perceived organizational support is, based on a survey of 401 Chinese post-90s employees. On the other hand, role pressures (including role overload, role blurring, role conflict) (Rhoades et al., 2002; Allen, 2008), organizational political activities (Hochwarter, 2003; Eisenberger \& Stinglhamber, 2011), organizational size (Rhoades et al., 2002) had negative impact on perceived organizational support. Ren et al. (2018) stated that organizational career management had a positive impact on perceived organizational support, through an empirical research of 362 knowledge employees in Zhejiang province, China. 


\subsubsection{Individual Factors}

Liao et al. (2004) found that differences of ethnicity, amenity and openness had negative predictive of perceived organizational support. Hui et al. (2007) showed that employees' traditional values and positive emotions were significantly related to perceived organizational support. Watt and Hargis (2010) found that workers who had tendency to be tired were not aware of the organization's support for them. Eisenberger and Stinglhamber (2011) stated that the antecedent variables of perceived organizational support included pre-employment experience, job status, and employee characteristics. In addition, Rhoades et al. (2002) confirmed that gender, age, education, and tenure in demographic variables had little effect on perceived organizational support.

\subsubsection{Employee-organization Relationship}

Sluss et al. (2008) stated that the leader-member exchange was positively related to the perceived organizational support of the subordinates. Zagenczyk et al. (2010) stated that colleagues would directly (through cohesive friendship and constructive contacts) and indirectly (through the position of the speech and friendship network structure) affect the employees' sense of organizational support. Eisenberger and Stinglhamber (2011) pointed out that leadership style, management communication, internal social network within the organization were closely related to perceived organizational support. Yang et al. (2011) found that interactive fairness had a positive impact on perceived organizational support using 520 female managers as a sample. Shoss et al. (2013) proved that abusive management led to a lower sense of organizational support from employees and their retaliation. A longitudinal study by Kiewitz et al. (2009) showed that when workers were aware of contract violations (that is, the organization fails to fulfill its obligations, employees believe they are in arrears), employees would think that the organization should be responsible for breaching the contract, resulting in a lower sense of organizational support. Mallette (2011) found that relationship contracts were also positively related to the sense of organizational support, while transactional contracts were negatively related to perceived organizational support. Empirical study of Tan (2012) showed that value match between individuals and the organization had a significantly positive influence on perceived organizational support.

Table 1. Antecedents of perceived organizational support

\begin{tabular}{|l|l|l|}
\hline Authors & Antecedents & Categories \\
\hline Wayne et al. & $\begin{array}{l}\text { organizational justice, } \\
\text { managerial tolerance, } \\
\text { recognition }\end{array}$ & organizational factors \\
\hline $\begin{array}{l}\text { Rhoades et al. } \\
(2002)\end{array}$ & $\begin{array}{l}\text { role pressures, } \\
\text { organizational size }\end{array}$ & organizational factors \\
\hline
\end{tabular}


Table 1. (continued)

\begin{tabular}{|c|c|c|}
\hline Authors & Antecedents & Categories \\
\hline $\begin{array}{l}\text { Hochwarter } \quad \text { (2003); } \\
\text { Eisenberger } \quad \& \\
\text { Stinglhamber (2011) }\end{array}$ & $\begin{array}{l}\text { organizational political } \\
\text { activities }\end{array}$ & organizational factors \\
\hline $\begin{array}{l}\text { Liao et al. } \\
\text { (2004) }\end{array}$ & $\begin{array}{l}\text { ethnicity, amenity, } \\
\text { openness }\end{array}$ & individual factors \\
\hline $\begin{array}{l}\text { Ling et al. } \\
(2006)\end{array}$ & procedural fairness & organizational factors \\
\hline $\begin{array}{l}\text { Hui et al. } \\
\text { (2007) }\end{array}$ & $\begin{array}{l}\text { employees' traditional } \\
\text { values, positive emotions }\end{array}$ & individual factors \\
\hline $\begin{array}{l}\text { Allen } \\
(2008)\end{array}$ & role pressures & organizational factors \\
\hline $\begin{array}{l}\text { Klimcak \& Holmes } \\
\text { (2008) }\end{array}$ & leader-member exchange & $\begin{array}{l}\text { employee-organization } \\
\text { relationship }\end{array}$ \\
\hline $\begin{array}{l}\text { Ghani } \\
(2009)\end{array}$ & $\begin{array}{l}\text { trust, access to } \\
\text { information, learning and } \\
\text { development opportunities }\end{array}$ & organizational factors \\
\hline $\begin{array}{l}\text { Zagenczyk et al. } \\
\text { (2010) }\end{array}$ & $\begin{array}{l}\text { friendship and contact } \\
\text { among colleagues }\end{array}$ & organizational factors \\
\hline $\begin{array}{l}\text { DeConinck } \\
(2010)\end{array}$ & distributive fairness & organizational factors \\
\hline $\begin{array}{l}\text { Dawley et al. } \\
\text { (2010) }\end{array}$ & $\begin{array}{l}\text { perceived supervisory } \\
\text { support }\end{array}$ & organizational factors \\
\hline $\begin{array}{l}\text { Watt \& Hargis } \\
(2010)\end{array}$ & tendency to be tired & individual factors \\
\hline
\end{tabular}




\section{Macrothink}

International Journal of Human Resource Studies

ISSN 2162-3058 2019, Vol. 9, No. 3

Table 1. (continued)

\begin{tabular}{|c|c|c|c|}
\hline Authors & & Antecedents & Categories \\
\hline \multirow{2}{*}{$\begin{array}{l}\text { Eisenberger } \\
\text { Stinglhamber } \\
\text { (2011) }\end{array}$} & \multirow{2}{*}{$\&$} & $\begin{array}{l}\text { pre-employment } \\
\text { experience, job status, } \\
\text { employee characteristics }\end{array}$ & individual factors \\
\hline & & $\begin{array}{l}\text { leadership style, } \\
\text { management } \\
\text { communication, internal } \\
\text { social network within the } \\
\text { organization }\end{array}$ & $\begin{array}{l}\text { employee-organization } \\
\text { relationship }\end{array}$ \\
\hline $\begin{array}{l}\text { Yang et al. } \\
(2011)\end{array}$ & & interactional fairness & organizational factors \\
\hline $\begin{array}{l}\text { Ahmed \& Nawaz } \\
(2015)\end{array}$ & & $\begin{array}{lr}\text { fairness, } & \text { growth } \\
\text { opportunities, supervisory } \\
\text { support, and colleague } \\
\text { support }\end{array}$ & organizational factors \\
\hline $\begin{array}{l}\text { Kiewitz et al. } \\
\text { (2009) }\end{array}$ & & $\begin{array}{l}\text { perceived contract } \\
\text { violations }\end{array}$ & $\begin{array}{l}\text { employee-organization } \\
\text { relationship }\end{array}$ \\
\hline $\begin{array}{l}\text { Mallette } \\
(2011)\end{array}$ & & $\begin{array}{l}\text { relationship contracts, } \\
\text { transactional contracts }\end{array}$ & $\begin{array}{l}\text { employee-organization } \\
\text { relationship }\end{array}$ \\
\hline $\begin{array}{l}\text { Tan } \\
(2012)\end{array}$ & & $\begin{array}{l}\text { values match between } \\
\text { individuals and the } \\
\text { organization }\end{array}$ & $\begin{array}{l}\text { employee-organization } \\
\text { relationship }\end{array}$ \\
\hline $\begin{array}{l}\text { Shoss et al. } \\
\text { (2013) }\end{array}$ & & abusive management & $\begin{array}{l}\text { employee-organization } \\
\text { relationship }\end{array}$ \\
\hline $\begin{array}{l}\text { Xue } \\
\text { (2017) }\end{array}$ & & $\begin{array}{l}\text { perceived organizational } \\
\text { justice }\end{array}$ & organizational factors \\
\hline
\end{tabular}


Table 1. (continued)

\begin{tabular}{|l|l|l|}
\hline Authors & Antecedents & Categories \\
\hline $\begin{array}{l}\text { Chen et al. } \\
(2017)\end{array}$ & $\begin{array}{l}\text { supportive human resource } \\
\text { practices }\end{array}$ & organizational factors \\
\hline Yu et al. & visionary leadership & organizational factors \\
$(2017)$ & $\begin{array}{l}\text { developmental human } \\
\text { desource practices (career } \\
\text { Lin } \\
\text { performance evaluation) }\end{array}$ & organizational factors \\
\hline $\begin{array}{l}\text { Zhang } \\
(2018)\end{array}$ & $\begin{array}{l}\text { organizational } \\
\text { socialization strategy }\end{array}$ & organizational factors \\
\hline Ren et al. & $\begin{array}{l}\text { organizational career } \\
\text { management }\end{array}$ & organizational factors \\
\hline
\end{tabular}

\subsection{Outcome Variables of Perceived Organizational Support}

There is an abundant research on outcome variables of perceived organizational support. The literature analysis showed that the outcome variables of perceived organizational support could be divided into job attitudes, job behaviors, and organizational performance (see Table 2). Among them, attitude-related variables include affective commitment, job satisfaction, positive emotions, and organizational trust and willingness to stay or leave; behavior-related variables include organizational citizenship behavior, and turnover behavior, and withdrawal behavior; organizational performance includes relationship performance and task performance.

\subsubsection{Job Attitudes}

Scholars have found, through empirical research, that perceived organizational support had positive influence on organizational commitment (Wayne et al., 2002; Wu, 2006), affective commitments (Rhoades et al., 2002; Ling et al., 2006; Lee \& Peccei, 2011), professional commitment (Tang \& Hu, 2017), job satisfaction (Rhoades et al., 2002; Stamper \& Johlke, 2003; McCarthy et al., 2013), stay intention (Rhoades et al., 2002; Stamper \& Johlke, 2003; Lee et al., 2011). Sense of organization support also had positive relationship with employees' positive emotion (Rhoades et al., 2002), organizational trust (Chen et al., 2005; 
Liu, 2018), organization-based self-esteem (Chen et al., 2005), organizational identity (Edwards, 2009; Fu et al., 2010; Wang \& Sun, 2011; He \& Pham, 2014). The sense of organizational support can also effectively reduce employee turnover tendency (Rhoades et al., 2002; Hui et al., 2007; Riggle et al., 2009; Dawley et al., 2010; McCarthy et al., 2013; Joo et al., 2015), relieve role pressures (such as role blurring and conflicts) (Rhoades et al., 2002; Stamper \& Johlke, 2003; McCarthy et al., 2013).

Wang et al. (2017) verified the positive correlation between perceived organizational support and perceived professional benefits through a survey questionnaire of 444 nurses of 17 hospitals in Hunan province of China. Sun (2017) stated that perceived organizational support has a significantly positive correlation with psychological contracts and has a significantly negative correlation with work burnout, through a survey of employees of 18 state-owned enterprises in Shandong province, China. Zhao (2018) stated that perceived organizational support has a negative correlation with work burnout of employees in the civil aviation industry by using Chinese civil aviation airport staff as a sample. Xu et al. (2017) verified that perceived organizational support has a significantly positive effect on perceived insider status through case studies. Chang et al. (2018) stated that perceived organizational support had a significantly positive predictive effect on psychological capital and career success of clinical nurses, through an empirical research on 794 clinical nurses of Liaoning province, China. Li et al. (2018) stated that perceived organizational support has a positive correlation with organizational identity, and with three dimensions (vigor, dedication, absorption) of work engagement, through a survey of 650 non-system workers of 6 public hospitals in Sichuan province, China. Wang, Q. (2018) stated that perceived organizational support of higher education teachers had a predictive effect on career adaptation and job satisfaction, based on an empirical study of 1410 teachers of 14 Chinese higher vocational colleges. Wei and Mao (2018) verified that perceived organizational support had a significantly positive impact on regulatory emotional self-efficacy using 274 research staff as a sample. Gao and Yan (2018) perceived organizational support had a significantly positive impact on entrepreneurial passion of science and technology personnel to leave their jobs through a survey of 297 research staff in Beijing, China.

\subsubsection{Job Behaviors}

The sense of organizational support had a positive impact on employees' performance outside the role and organizational citizenship behavior, at the same time, could reduce employee negative behaviors, such as resignation, anti-productive behavior, withdrawal behavior (negative absenteeism, being late, absence). For example, George and Brief (1992) stated that the perception of organizational support enabled employees to generate some organizational citizenship behaviors, such as help organizations avoid risks, provide constructive advice, help colleagues, etc. Wayne et al. (2002) found that perceived organizational support was negatively related to negative behaviors in the organization (negative sabotage, absenteeism). On the other hand, there are some researches which stated positive predictive effects of perceived organizational support. For example, Chen et al. (2005) discussed the predictive effect of perceived organizational support on positive variables, such as organizational commitment and organizational citizenship behavior. Piercy et al. (2006), in sales staff 
studies, found that perceived organizational support had a strong influence on organizational citizenship behavior. Lee and Peccei (2011) research showed that perceived organizational support could predict individual-oriented organizational citizenship behavior. Zheng et al. (2017) found out that perceived organizational support of medical staff was closely related to organizational citizenship behavior through a survey of 600 medical staff in 6 medical institutions of Shijiazhuang, China. Yu et al. (2017) stated perceived organizational support had an impact on voice behavior of employees. Zheng and Hu (2018) made an empirical research on the relationship between perceived organizational support and innovative deviance with 400 employees in internet industry as the sample, their findings showed that job support and care about interests have significantly positive influence on innovative deviance, while value recognition had no significant influence on innovative deviance based on Ling et al.'s (2006) three-dimension POS structure. Wang, C. L. (2018) stated that perceived organizational support could have a significantly positive influence on employee communication intention and innovation behavior through an empirical study. Li (2018) stated that perceived organizational support had a significantly positive correlation with job adaptation and self-career management of new employees through a survey questionnaire for employees who have worked for less than one year.

\subsubsection{Organizational Performance}

Kraimer and Wayne (2004) studied the effects of perceived organizational support, leader-member exchange, and spouse support on job adaptation and job performance of expatriate employees. The research results showed that organizational support had a direct impact on the adaptation of expatriate employees, which in turn affected the employee's task performance and relationship performance. Although the leader-member exchange had no obvious impact on overseas adaptation, it had a direct impact on task performance and relationship performance. Spouse support had no significant effect on overseas adaptation and job performance. The research of Chong et al. (2001) on production staff showed that employees with higher perception of organizational support had a more positive attitude towards just-in-time (JIT) management, and the improvement of work performance was more obvious. A study by Bell and Menguc (2002) on sales personnel showed that the value of customer evaluations for the quality of service of employees with good organizational support was relatively high. In these studies, researchers mostly used organizational commitment, organizational identity, job satisfaction, and other factors as mediating factors impacting job performance. Meta-analysis of Rhoades et al. (2002) showed that the sense of organizational support was moderately related to the out-of-role performance of the organization. Chen et al.'s (2009) longitudinal study confirmed that the sense of organizational support led to out-of-role performance. Eisenberger and Stinglhamber (2011) stated that the results of positive behaviors of organizational support help organizations achieve their goals. These positive behavioral outcomes include reduction of withdrawal behavior and workplace deviant behavior, creativity and innovation, more safety-related activities, and easier acceptance of information technologies, increased customer service. Shoss et al. (2013) found that a low sense of organizational support increased employees' anti-productive behavior and reduced intra-role performance and out-of-role performance. 


\section{Macrothink}

International Journal of Human Resource Studies

ISSN 2162-3058 2019, Vol. 9, No. 3

Kurtessis et al.'s (2017) meta-analysis showed that the outcome variables of perceived organizational support mainly included employee service to organization and work, employee performance and happiness.

Table 2. Outcomes of perceived organizational support

\begin{tabular}{|c|c|c|}
\hline Authors & Outcomes & Categories \\
\hline \multirow[t]{2}{*}{$\begin{array}{l}\text { Wayne et al. } \\
\text { (2002) }\end{array}$} & $\begin{array}{l}\text { organizational } \\
\text { commitment }\end{array}$ & employee attitude \\
\hline & negative behaviors & employee behavior \\
\hline \multirow[t]{2}{*}{$\begin{array}{l}\text { Rhoades et al. } \\
\text { (2002) }\end{array}$} & $\begin{array}{l}\text { affective commitments, } \\
\text { job satisfaction, stay } \\
\text { intention, employees } \\
\text { positive } \\
\text { employee emotion, } \\
\text { tendency, role pressures }\end{array}$ & employee attitude \\
\hline & $\begin{array}{l}\text { out-of-role performance of } \\
\text { the organization }\end{array}$ & organizational performance \\
\hline $\begin{array}{l}\text { Eisenberger } \\
\text { Stinglhamber } \\
(2011)\end{array}$ & $\begin{array}{l}\text { withdrawal behavior, } \\
\text { workplace deviant } \\
\text { behavior, creativity and } \\
\text { innovation, rafety, } \\
\text { acceptance of information } \\
\text { technologies, customer } \\
\text { service }\end{array}$ & organizational performance \\
\hline $\begin{array}{l}\text { George \& Brief (1992) } \\
\text { Piercy et al. (2006) } \\
\text { Lee \& Peccei (2011) } \\
\text { Zheng et al. (2017) }\end{array}$ & $\begin{array}{l}\text { organizational citizenship } \\
\text { behavior }\end{array}$ & employee behavior \\
\hline $\begin{array}{l}\text { Chong et al. } \\
\text { (2001) }\end{array}$ & $\begin{array}{l}\text { just-in-time management, } \\
\text { work performance }\end{array}$ & organizational performance \\
\hline $\begin{array}{l}\text { Bell \& Menguc } \\
(2002)\end{array}$ & customer evaluations & organizational performance \\
\hline
\end{tabular}


Table 2. (continued)

\begin{tabular}{|c|c|c|}
\hline Authors & Outcomes & Categories \\
\hline $\begin{array}{l}\text { Kraimer \& Wayne } \\
\text { (2004) }\end{array}$ & $\begin{array}{l}\text { job adaptation, task } \\
\text { performance, relationship } \\
\text { performance }\end{array}$ & organizational performance \\
\hline \multirow[t]{2}{*}{$\begin{array}{l}\text { Chen et al. (2005) } \\
\text { Liu (2018) }\end{array}$} & $\begin{array}{l}\text { organizational trust, } \\
\text { organization-based } \\
\text { self-esteem, organizational } \\
\text { commitment }\end{array}$ & employee attitude \\
\hline & $\begin{array}{l}\text { organizational citizenship } \\
\text { behavior }\end{array}$ & employee behavior \\
\hline $\begin{array}{l}\text { Edwards (2009) } \\
\text { Fu et al. (2010) } \\
\text { Wang \& Sun (2011) } \\
\text { He \& Pham (2014) } \\
\text { Li et al. (2018) }\end{array}$ & organizational identity & employee attitude \\
\hline $\begin{array}{l}\text { Shoss et al. } \\
(2013)\end{array}$ & $\begin{array}{l}\text { anti-productive behavior, } \\
\text { intra-role performance and } \\
\text { out-of-role performance }\end{array}$ & organizational performance \\
\hline $\begin{array}{l}\text { Tang and } \mathrm{Hu} \\
\text { (2013) }\end{array}$ & professional commitment & employee attitude \\
\hline $\begin{array}{l}\text { Sun } \\
(2017)\end{array}$ & $\begin{array}{l}\text { psychological contracts, } \\
\text { work burnout }\end{array}$ & employee attitude \\
\hline $\begin{array}{l}\text { Kurtessis et al. } \\
\text { (2017) }\end{array}$ & $\begin{array}{l}\text { employee service to } \\
\text { organization and work, } \\
\text { employee performance, } \\
\text { employee happiness }\end{array}$ & organizational performance \\
\hline $\begin{array}{l}\text { Xu et al. } \\
\text { (2017) }\end{array}$ & perceived insider status & employee attitude \\
\hline
\end{tabular}


Table 2. (continued)

\begin{tabular}{|c|c|c|}
\hline Authors & Outcomes & Categories \\
\hline $\begin{array}{l}\text { Wang et al. } \\
\text { (2017) }\end{array}$ & $\begin{array}{l}\text { perceived professional } \\
\text { benefits }\end{array}$ & employee attitude \\
\hline $\begin{array}{l}\text { Yu et al. } \\
\text { (2017) }\end{array}$ & voice behavior & employee behavior \\
\hline $\begin{array}{l}\text { Zheng \& Hu } \\
\text { (2018) }\end{array}$ & innovative deviance & employee behavior \\
\hline $\begin{array}{l}\text { Li et al. } \\
\text { (2018) }\end{array}$ & work engagement & employee attitude \\
\hline $\begin{array}{l}\text { Chang et al. } \\
\text { (2018). }\end{array}$ & $\begin{array}{l}\text { psychological capital, } \\
\text { career success }\end{array}$ & employee attitude \\
\hline $\begin{array}{l}\text { Wang, Q. } \\
\text { (2018) }\end{array}$ & $\begin{array}{l}\text { career adaptation, job } \\
\text { satisfaction }\end{array}$ & employee attitude \\
\hline $\begin{array}{l}\text { Wei \& Mao } \\
(2018)\end{array}$ & $\begin{array}{l}\text { regulatory emotional } \\
\text { self-efficacy }\end{array}$ & employee attitude \\
\hline $\begin{array}{l}\text { Zhao } \\
\text { (2018) }\end{array}$ & work burnout & employee attitude \\
\hline \multirow[t]{2}{*}{$\begin{array}{l}\text { Wang, C. L. } \\
(2018)\end{array}$} & $\begin{array}{l}\text { employee communication } \\
\text { intention }\end{array}$ & employee attitude \\
\hline & innovation behavior & employee behavior \\
\hline $\begin{array}{l}\text { Gao \& Yan } \\
(2018)\end{array}$ & entrepreneurial passion & employee attitude \\
\hline Li (2018) & self-career management & employee behavior \\
\hline
\end{tabular}




\section{Strategies of Optimizing Perceived Organizational Support}

Based on the principle of reciprocity, individuals have the responsibility and obligation to actively respond to the positive treatment of the other party. In the organization, employees will reciprocate the organization by adopting attitudes and behaviors expected by the organization and being committed to the organization when they feel the support and commitment from the organization. Thus, it's important for organizations to improve employee perception of organizational support. So far, there are a few articles which studied the strategies to optimize perceived organizational support. For instance, Hu et al. (2014) suggested that managers need to pay attention to three important sources of perceived organization support-procedural fairness at work, superior support, reward and supportive working conditions so as to make employees feel organizational value on their contributions and well-being, and produce higher organizational performance. Liu et al. (2015) stated that the organization should implement high commitment HR practices based on employee needs, and pay attention to openness and fairness of HRM information. Hakeem and Nisa (2016) provided some measures on how to enhance employee perception of organizational support from the aspects of supervisor support, procedural justice, coworker support, HR practices (equitable rewards, recognition of new ideas by employees, providing career development opportunities, training, job security, job autonomy and work-family support). Eisenberger et al. (2016) proposed eight tactics for optimizing perceived organizational support, respectively 1) Implement supportive workforce services that are discretionary. 2) Be fair and equitable in the making, monitoring and enforcement of all management practices. 3) Set achievable goals and reward proportionately. 4) Offer individualized benefits. 5) Support supervisors so they will foster POS in their subordinates. 6) Train subordinates to be supportive. 7) Promote strong social networks. 8) Begin organizational support prior to the start of employment. Chen et al. (2017) suggested to improve employee perception of organizational support from supportive HR practices such as learning employees' expectation, creating fair environment, implanting continuous HR practices, and also to shape the sense of ownership of employees to promote colleague support in organizations. Zhang (2018) stated that the organization could give proper empowerment to employees and job security during economic recession so as to increase employee trust in organization and perceived organizational support.

\section{Conclusion}

\subsection{Summary}

Overall, our findings contribute to the organizational psychology literature and human resource management literature. Perceived organizational support reflects the employee and organization relationship which is essentially a social exchange. This exchange includes both material exchange and non-material exchange. This research introduced the origin of perceived organizational support and its important benefits for employees and employers, distinguished perceived organizational support from psychological contracts, synthesized some important factors that positively relates perceived organizational support and positive outcomes that perceived organizational support leads to, finally reviewed some measures for optimizing perceived organizational support in recent studies. To sum up, perceived 
organizational support has changed the past one-side emphasis on employees' commitment to the organization. For the first time, the concept of perceived organizational support emphasized the importance of organization's commitment to employees (employers' commitments), filling the gap in HRM theories.

\subsection{Discussion}

Perceived organizational support can be influenced by all aspects of the organization's treatment of employees, which in turn will affect the employee's interpretation of the organization's potential motivation for treating employees. Organizational support includes the organization's possible response to the employee's future illnesses, errors, and high performance, as well as the organization's willingness to pay a fair salary to make the employee's work meaningful and interesting. In addition, the role of perceived organizational support in promoting positive employee attitudes, behavior and performance indicates two phenomena. One is employees need organization's support for the sake of both benefits of employees and employers. Organizational support is one of necessary needs in today's business, because employees may gradually exhaust their energy backup and might eventually burn out when job demands were high, additional effort must be exerted to achieve the work goals, and when recovery was inadequate or insufficient (Schaufeli, 2013). The other is employees tend to reward organizational positive treatment for the sake of mutual interests of employees and employers. Based on the principle of reciprocity, when they feel the beneficial treatment of the organization, employees will have a general obligation to organize and help the organization to achieve its goals, and thus demonstrate an attitude and behavior conducive to the organization. Finally, the strategies for optimizing perceived organizational support link the employee-organizational relationship to supportive HR practices. Since employee perception of organizational support is rather important in influencing their attributions to organizational behaviors and then guide their job attitudes and behavior, HR department needs to understand the measures which can improve employee perception of organizational support, formulate organizational support program before employment, constantly monitor the level of perceived organizational support of employees and take corrective actions when needed, so as to make sure favorable organizational outcomes.

\subsection{Limitations and Suggestions for Future Research}

This research has some limitations. First, this research only summarized the antecedents and outcomes of perceived organizational support, and lacked the review of perceived organizational support as a mediator variable or moderator variable. Besides, this research lacked the review of perceived organizational support for diversified employees (gender, race, education, position), because diverse employees have different expectations and perceptions of organizational support. Moreover, employees in different industries also have different needs and feedback for organizational support. Therefore, future research needs to collect the literature about the mediating or moderating effect of perceived organizational support between variables. Besides, future research can collect and summarize the research findings related to perceived organizational support for a certain type of employee, such as gender, age, 
race, education, position, working period, industry, etc.

\section{References}

Ahmed, I., \& Nawaz, M. M. (2015). Antecedents and outcomes of perceived organizational support: A literature survey approach. Journal of management development, 34(7), 867-880. https:// doi.org/10.1108/JMD-09-2013-0115

Allen, M. W. (2008). Factors impacting the perceived organizational support of it employees. Information \& management, 45(8), 556-563. https://doi.org/10.1016/j.im.2008.09.003

Aselage, J., \& Eisenberger, R. (2003). Perceived organizational support and psychological contract: A theoretical integration. Journal of organizational behavior, 24(5), 491-509.

https:// doi.org/10.1002/job.211

Becker, G. S. (2002). The age of human capital. Retrieved from http://media.hoover.org/sites/default/files/documents/0817928928_3.pdf.

Bell, S. J., \& Menguc, B. (2002). The Employee-organization relationship, organizational citizenship behaviors, and superior service quality. Journal of retailing, 78(2), 131-146. https://doi.org/10.1016/S0022-4359(02)00069-6

Chang, X. Y., Ma, Y. M., Mao, X., \& Chu, Y. (2018). Analysis of the mediating role of psychological capital between perceived organizational support and career success of clinical nursing staff. China health statistics, 35(2), 259-266.

Chen, J. A., Cheng, S., \& Chen, M. Y. (2017). A study on the internal mechanism of supportive human resource practices shaping perceived organizational support. Chinese journal of management, 14(4), 72-83. https://doi.org/10.3969/j.issn.1672-884x.2017.04.006

Chen, Z. X., Aryee, S., \& Lee, C. (2005). Test of a mediation model of perceived organizational support. Journal of vocational behavior, 66(3), 457-470.

https://doi.org/10.1016/j.jvb.2004.01.001

Chen, Z. X. (2006). The effect of sense of organizational support of knowledge employees on job performance and turnover intention (Unpublished doctoral dissertation). Huazhong University of Science and Technology, China.

Chen, Z., Eisenberger, R., Johnson, K. M., Sucharski, I. L., \& Aselage, J. (2009). Perceived organizational support and extra-role performance: Which leads to which? Journal of social psychology, 149(1), 119-124. https://doi.org/10.3200/SOCP.149.1.119-124 •

Chong, H., White, R. E., \& Prybutok, V. (2001). Relationship among organizational support, JIT implementation, and performance. Industrial management and data systems, 101(5/6), 273-280. https:// doi.org/10.1108/EUM0000000005576

Dawley, D., Houghton, J., \& Bucklew, N. (2010). Perceived organizational support and turnover intention: The mediating effects of personal sacrifice and job fit. Journal of social psychology, 150(3), 238-257. https:// doi.org/10.1080/00224540903365463 
DeConinck, J. (2010). The effect of organizational justice, perceived organizational support, and perceived supervisor support on marketing employees' level of trust. Journal of business research, 63(12), 1349-1355. https://doi.org/10.1016/j.jbusres.2010.01.003

Edwards, M. (2009). HR, perceived organizational support and organizational identification: An analysis after organizational formation. Human resource management journal, 19(1), 91-115. https://doi.org/10.1111/j.1748-8583.2008.00083.x

Eisenberger, R., Huntington, R., Hutchison, S., \& Sowa, D. (1986). Perceived organizational support. Journal of applied psychology, 71(3), 500-507.

http://dx.doi.org/10.1037/0021-9010.71.3.500

Eisenberger, R., Cummings, J., Armeli, S., \& Lynch, P. (1997). Perceived organizational support, discretionary treatment and job satisfaction. Journal of applied psychology, 82(5), 812-820. https:// doi.org/10.1037/0021-9010.82.5.812

Eisenberger, R., \& Stinglhamber, F. (2011). Perceived organizational support: Fostering enthusiastic and productive employees. Washington, DC, US: American Psychological Association.

Eisenberger, R., Malone, G. P., \& Presson, W. D. (2016). Optimizing perceived organizational support to enhance employee engagement. SHRM-SIOP Science of HR series, 1-22. Retrieved from

https://www.shrm.org/hr-today/trends-and-forecasting/special-reports-and-expert-views/Docu ments/SHRM-SIOP\%20Perceived\%20Organizational\%20Support.pdf

Fu, S., Ding, N. N., \& Zhao, Y. Q. (2010). Research on social exchange in enterprises: Perceived organizational support and leadership support. Science and technology management, 31(6), 175-181.

Gao, J. L., \& Yan, M. (2018). The impact of perceived organizational support on research personnel's entrepreneurial passion-Psychological capital as mediator variable. Technical economics and management research, 7, 33-37.

George, J. M., \& Brief, A. P. (1992). Feeling good-doing well: A conceptual analysis of the mood at work-organizational spontaneity relationship. Psychology bulletin, 112, 310-329.

Ghani, N. A. (2009). Antecedents of perceived organizational support. Canadian social science, 5(6), 33-42.

Hakeem, I. A., \& Nisa, N. U. (2016). Perceived organizational support: A review on its antecedents and outcomes. Journal of research in management \& technology, 5(6), 30-38.

He, H., \& Pham, H. Q. (2014). Perceived organizational support and organizational identification: Joint moderating effects of employee exchange ideology and employee investment. International journal of human resource management, 25(20), 2772-2795.

https://doi.org/10.1080/09585192.2014.908315

Hochwarter, W. A. (2003). Perceived organizational support as a mediator of the relationship 
between politics perceptions and work outcomes. Journal of vocational behavior, 63(3), 438-456. http://dx.doi.org/10.1016/S0001-8791(02)00048-9

Hu, S. M., Liu, M. Y., \& Zhang, X. Q. (2014). The moderating effect of perceived organizational support and the impact of job insecurity on OCB. Journal of Huaqiao university (philosophy \& social sciences), 3, 72-83.

Hui, C., Wong, A., \& Tjosvold, D. (2007). Turnover intention and performance in China: The role of positive affectivity, Chinese values, perceived organizational support and constructive controversy. Journal of occupational and organizational psychology, 80(4), 735-751.

https:// doi.org/10.1348/096317906X171037

Kiewitz, C., Restubog, S. L. D., Zagenczyk, T., \& Hochwarter, W. (2009). The interactive effects of psychological contract breach and organizational politics on perceived organizational support: Evidence from two longitudinal studies. Journal of management studies, 46(5), 806-834. https:// doi.org/10.1111/j.1467-6486.2008.00816.x

Kraimer, M. L., \& Wayne, S. J. (2004). An examination of perceived organizational support as a multidimensional construct in the context of an expatriate assignment. Journal of management, 30(2), 209-237. https://doi.org/10.1016/j.jm.2003.01.001

Kurtessis, J. N., Eisenberger, R., Ford, M. T., Buffardi, L. C., Stewart, K. A., \& Adis, C. S. (2017). Perceived organizational support: A meta-analytic evaluation of organizational support theory. Journal of management, 43(6), 1854-1884.

https:// doi.org/10.1177/0149206315575554

Lee, J., \& Peccei, R. (2011). Discriminant validity and interaction between perceived organizational support and perceptions of organizational politics: A temporal analysis. Journal of occupational and organizational psychology, 84(4), 686-702.

https://doi.org/10.1348/096317910X511197

Li, D. Y. (2018). The impact of organizational support on job adaptation of new employees-The mediating role of self-care management. China management informationization, 21(7), 95-96. https://doi.org/10. 3969/j. issn. 1673 - 0194. 2018. 07. 039

Lin, N. N. (2018). Research on the relationships among developmental human resources practices, perceived organizational support and turnover intention of knowledge workers in enterprises (Unpublished master's thesis). Hebei University of Economics and Business, China.

Ling, W. Q., Yang, H. J., \& Fang, L. L. (2006). Perceived organizational support of the employees. Journal of psychology, 38(2), 281-287.

Liu, S. Y. (2018). The study of impact of perceived organizational support on job involvement-The mediation effect of organizational trust (Unpublished master's thesis). Guangxi University, China.

Liu, Z. H., Li, Y. P., \& Mao, T. P. (2015). High commitment human resource practices and employee performance-The mediating effect of perceived organizational support. Soft 
science, 29(10), 92-100. https:// org/10.13956/j.ss.1001-8409.2015.10.20

Mallette, C. (2011). Nurses' work patterns: Perceived organizational support and psychological contracts. Journal of research in nursing, 16(6), 518-532.

https://doi.org/10.1177/1744987111422421

McCarthy, A., Cleveland, J. N., Hunter, S., Darcy, C., \& Grady, G. (2013). Employee work-life balance outcomes in Ireland: A multilevel investigation of supervisory support and perceived organizational support. International journal of human resource management, 24(6), 1257-1276. https://doi.org/10.1080/09585192.2012.709189

McMillin, R. (1997). Customer satisfaction and organizational support for service providers (Unpublished doctoral dissertation). University of Florida, Gainesville.

Piercy, N. (2006). Driving organizational citizenship behaviors and salesperson in-role behavior performance: The role of management control and perceived organizational support. Journal of the academy of marketing science, 34(2), 244-262.

http://dx.doi.org/10.1177/0092070305280532

Ren, Z. J., Tao, R. S., \& Hu, Z. H. (2018). Impact of organization career management on career growth of knowledge employee: Mediation effect of perceived organizational support. Journal of Hubei university of arts and science, 39(5), 48-52.

Rhoades, L., \& Eisenberger, R. (2002). Perceived organizational support: A review of the literature. Journal of applied psychology, 87(4), 698-714.

http://dx.doi.org/10.1037/0021-9010.87.4.698

Riggle, R., Edmondson, D., \& Hansen, J. (2009). A meta-analysis of the relationship between perceived organizational support and job outcomes: 20 years of research. Journal of business research, 62(10), 1027-1030. https://doi.org/10.1016/j.jbusres.2008.05.003

Schaufeli, W. B. (2013). What is engagement? In C. Truss, K. Alfes, R. Delbridge, A. Shantz, \& E. Soane (Eds.), Employee engagement in theory and practice (pp. 16-17). London: Routledge.

Schermerhorn, J. R. (2010). Introduction to management (10th ed.). Asia: Wiley.

Shoss, M., Eisenberger, R., Restubog, S., \& Zagenczyk, T. (2013). Blaming the organization for abusive supervision: The roles of perceived organizational support and supervisor's organizational embodiment. Journal of applied psychology, 98(1), 158-168.

https://doi.org/10.1037/a0030687

Sluss, D. M., Klimcak, M., \& Holmes, J. J. (2008). Perceived organizational support as a mediator between rational exchange and organizational identification. Journal of vocational behavior, 73(3), 457-464. https://doi.org/10.1016/j.jvb.2008.09.001

Stamper, C., \& Johnlke, M. (2003). The impact of perceived organizational support on the relationship between boundary spanner role stress and work outcomes. Journal of management, 29(4), 569-588. https:// doi.org/10.1016/S0149-2063(03)00025-4 
Sun, X. (2017). Research on relationship between perceived organizational support, psychological contract and work burnout: Taking state-owned enterprises in Shandong province as an example (Unpublished master's thesis). Guizhou University of Finance and Economics, China.

Tan, X. H. (2012). The influence of matching personal and organizational values on employees' work engagement and perceived organizational support. Psychological science, 35(4), 937-977. http://dx.doi.org/10.16719/j.cnki.1671-6981.2012.04.015

Tang, J. B., \& Hu, E. H. (2017). Research on the effect of perceived organizational support on the job satisfaction of middle school teachers. Mathematics in practice and theory, 47(24), 307-318.

Wang, C. L. (2018). Research on the relationship between perceived organizational support, employee communication intention and innovation behavior (Unpublished master's thesis). Tianjin Polytechnic University, China.

Wang, D. H., Yue, J. H., Wu, W. S., Gong, Y. L., \& Zhong, J. (2017). Relationships between perceived organizational support and perceived professional benefits of nurses in grade 2 hospitals. Journal of nursing science, 32(8), 69-95.

https://doi.org/10.3870/j.issn.1001-4152.2017.08.069

Wang, Q. (2018). Research on the relationship between teachers' organizational support and job satisfaction in higher vocational colleges-Mediating role of professional adaptation. Chinese higher education research, 9, 104-108.

https://doi.org/10.16298/j.cnki.1004-3667.2018.09.19

Wang, Z., \& Sun, J. M. (2011). Human resource management practice, organizational support, employee commitment and identity: A cross-level study. Economic management, 4, 80-86. http://doi.org/10.19616/j.cnki.bmj.2011.04.013

Watt, J. D., \& Hargis, M. B. (2010). Boredom proneness: Its relationship with subjective underdevelopment, perceived organizational support, and job performance. Journal of business \& psychology, 25(1), 163-174. https:// doi.org/10.1007/s10869-009-9138-9

Wayne, S., Shore, L. M., Bommer, W. H., \& Tetrick, L. E. (2002). The role of fair treatment and rewards in perceptions of organizational support and leader-member exchange. Journal of Applied psychology, 87(3), 590-598.

Wei, L. Y., \& Mao, T. T. (2018). Study on the relationship between perceived organizational support, regulatory emotional self-efficacy and employee well-being. Economic research guide, 13, 85-90.

$\mathrm{Wu}$, J. H. (2006). Empirical research on the influence of organizational support cognition and leadership-member exchange on employee return. Soft science, 20(5), 63-66.

Xu, B. H., \& Zhang, X. G. (2005). Progress in perceived organizational support. Applied psychology, 11(4), 325-329. 


\section{Macrothink}

International Journal of Human Resource Studies

ISSN 2162-3058 2019, Vol. 9, No. 3

Xu, J., Zhao, L., Wei, L. H., \& Shen, Y. M. (2017). The impact of perceived organizational support on organizational identification: The mediating effects of perceived insider status and organization-based self-esteem. Psychological exploration, 37(3), 275-282.

Xue, Q. (2017). The study of influence of perceived organizational justice on "post-90s" employees' job involvement: The mediating role of perceived organizational support (Unpublished master's thesis). Anhui University of Science and Technology, China.

Yang, F., Zhang, L. H., \& Huo, M. (2011). Can interactive fairness really arouse the responsibility of female managers in China? The mediating role of perceived organizational support. Economic science, 6, 117-128.

Yu, K., Liu, X. Y., \& Huang, Y. H. (2017). Research on the influence of visionary leadership on employees' voice behavior: The role of organizational support and challenge-based stress assessment. Modern management science, 5, 106-108.

Yuan, P. P. (2017). Research on post-90s staff's turnover intention based on perceived organizational (Unpublished master's thesis). Chongqing Jiaotong University, China.

Zagenczyk, T. J., Scott, K. D., Gibney, R., Murrell, A. J., \& Thatcher, J. B. (2010). Social influence and perceived organizational support: A social networks analysis. Organizational behavior and human decision processes, 111(2), 127-138.

http://dx.doi.org/10.1016/j.obhdp.2009.11.004

Zhang, N. (2018). Empirical study on influence of organizational socialization strategy, perceived organizational support upon employee turnover intention (Unpublished master's thesis). Shandong University, China.

Zhao, J. R. (2018). Organizational support, employee burnout and human factor risk management-Example of the civil aviation airport. Economic research guide, 22, 99-102.

Zheng, C. J., \& Hu, P. P. (2018). Research on the relationship between organizational support and Bootleg innovation in Internet firms-The mediating effect of job satisfaction. West forum on economy and management, 29(2), 72-80.

Zheng, S. Y., Li, S. R., Yang, H. X., \& Lv, M. N. (2017). Study on the relationship between perceived organizational support and organizational citizenship behavior of medical staff in Shijiazhuang. Medicine and society, 30(11), 43-46.

https:// doi.org/1.13723/j.yxysh.2017.11.013

\section{Copyright Disclaimer}

Copyright for this article is retained by the author(s), with first publication rights granted to the journal.

This is an open-access article distributed under the terms and conditions of the Creative Commons Attribution license (http://creativecommons.org/licenses/by/4.0/). 